lecturer or by a group of students. Unlike several British books having similar titles, this is not a guide to precise experimental technique. The word "practical". in the title may perhaps be justified by saying that the lectures were designed to suit "practical men" in the sense of people of I.Q. about 120, who are familiar with tools, who have scant time for study, and who may be tired after a day's toil. Theory, such as occurs in first-year university courses, is here cut out as much as possible: there remains a little algebra and trigonometry, but no calculus. For example, a chapter on uniform circular motion begins with pictures of machines, states the formula for centripetal force without proof, and explains carefully its general interpretation and briefly its applications to aviation, to cream separators and to banked roadways. The chapter then offers a set of numerical examples, and ends with an experiment in which a girl student is shown holding a vertical glass tube through which passes a string, attached below to a weight, and above to a mass which she whirls in a horizontal circle. A blank form is provided for the observations and deductions.

Protest must be made against the reappearance (on p. 56) of the old fallacy that the time taken for a rise of temperature to move along a metal bar de. pends only on its conductance ; whereas, in fact, its specific heat and density are just as essential.

Honours graduates in physies, who are about to begin to lecture in National Certificate courses, would find in this book a guide to what is possible in those circumstances.

L. F. Richardion.

\section{Recent Advances in Galactic Dynamics}

By Prof. A. C. Banerji. (Lucknow University Studies, No. 15.) Pp. vi+116. (Lucknow : Lucknow University, 1942.)

$\mathrm{T}$ HE author of this book was invited to give three extension lectures at the University of Luck. now in 1940 on galactic dynamics, and they have now been published in the form of a monograph. The first lecture deals with polytropic configurations of rotating gaseous matter, and provides an excellent summary of the work of investigators from the earliest times up to the present. It ends with a brief discussion of the numerical solution of the differential equations which occur in the cases of polytropic configurations of rotating gases. The second lecture summarizes the investigations of Hubble, Jeans, Brown, Vogt and Lambrecht, Lindblad, and a number of Indian workers, including the author, on spiral nebulæ. A full account of the researches of Indian workers has appeared (Phil. Mag., 7, 28, 118 ; 1939), and also in a number of Indian publications of more recent date. The origin of the solar system is considered in the third lecture, and the various theories to explain it are dealt with, from Swedenborg and Kant's nebular hypothesis to the recent theory of Lyttleton that the sun was a binary, its companion being disrupted by a passing star, the planets and satellites being formed from the debris. The investigations of Bhatnagar are considered and these have an important bearing on certain problems connected with planetary formation. He has shown that there is no possibility of the formation of the ribbon, from which the planets were supposed to be formed, by a close encounter or even a grazing collision between stars of normal masses. In addition, he has shown that the origin of the solar system is inexplicable by any existing tidal theory.
This is an excellent summary of our knowledge of stellar dynamics, and its value is enhanced by the references to all the workers on this subject.

$$
\text { M. D. }
$$

Intermediate Qualitative Analysis (Inorganic)

By Dr. A. J. E. Welch. Pp. iv +154 . (London : University Tutorial Press, Ltd., 1943.) 3s. $9 d$.

$\mathrm{T}$ HIS book provides theoretical and practical instructions for the qualitative analysis of $\mathrm{mix}$ tures, and can be recommended for the use of students at the Intermediate and Higher Certificate stage.

An outline of the theoretical principles underlying qualitative analysis is given first of all, and, apart from the use of the obsolete ferric-thiocyanate equation for the illustration of the law of mass action, it is quite adequate. There then follows an excellent chapter on the preliminary tests, apparatus and experimental technique of analysis. Especially clear instructions are given on the use of the blowpipe, platinum wire, etc., in the dry tests which are so frequently bungled by inexperienced students.

The reactions for the identification of metallic radicals are described in sufficient detail, but it is a great pity that they have been represented through. out by molecular equations and the opportunity thereby lost of emphasizing the ionic character of most of them. The tests for acid radicals have been arranged in a system of three groups which should be easy to operate.

In the closing chapter the tests are summarized in a tabular form which should prove useful, especially as the correct quantities of the reagents have been specified.

A. C. C.

\section{A New German-English Psycho-Analytical Vocabu- lary}

By Alix Strachey. (Research Supplements to the International Journal of Psycho-Analysis, No. 1 : Published for the Institute of Psycho-Analysis.) Pp. $\mathrm{v}+84$. (London: Baillière, Tindall and Cox, 1943.) 10s. 6d. net.

DICTIONARIES are of use to many and a source of real joy to a few. To this small minority the present slim volume will prove additionally attractive through its excellent typography. It is, as the preface states, essentially an enlarged and revised edition of a glossary issued as a supplement to the International Journal of Psycho-analysis in 1924. It does not. contain the interesting notes and comments appended. to certain entries in the former work, but with the increasing standardization of psycho-analytic terminology in the intervening years these are now scarcely necessary. A good many terms that were being tentatively introduced at the earlier date have now been generally adopted; for example, 'parapraxis' and 'cathexis' (for Fehlleistung and Besetzung respectively). On the other hand, the word "unpleasure' (for Unlust), formerly described as "hardly acceptable linguistically", is now accorded the dignity of a technical term. Those who grumble at the 'Freudian jargon' will probably express 'displeasure' at such terms as 'scopophilia' and 'osphresiophilia', but would they stomach 'peeping' and 'sniffing' any better? It is perhaps a pity that Havelock Ellis's more euphonious 'eonism' (on analogy with 'sadism' and 'masochism') is not given as an alternative to 'transvestitism'.

J. C. Fluaks. 